\title{
EXACT PHASE AND ITS EIKONAL . APPROXIMATION FOR CLASS OF REFLECTIONLESS INDEX PROFILES
}

\author{
S.K. SHARMA \\ S.N. Bose National Centre for Basic Sciences \\ DB 17 Bidhar Nagar, Calcutta-700 064, India \\ AND G. GHOSH \\ Saha Institute of Nuclear Physics \\ 1/AF Bidhan Nagar, Calcutta-700 064, India
}

(Received July 31, 1996; revised version November 13, 1996)

We examine the problem of transmission of electromagnetic waves in a multiparameter reflectionless dielectric. It is shown that it is possible to obtain an analytic form for the phase of the exact transmission amplitude. In the high frequency limit the exact result is found to lead to the eikonal approximation. Interestingly, the high frequency limit and long-range approximation required for the validity of the eikonal approximation are not independent here.

PACS numbers: $41.20 . \mathrm{Jb}, 42.25 . \mathrm{Fx}$

\section{Introduction}

A very old and interesting problem in optics had been the specification of the refractive index profile of a dielectric slab which is perfectly transparent to propagation of electromagnetic waves. Here it is known for a long time that the idealized situation of an infinite dielectric, where the refractive index varies continuously, admits an infinite number of profiles with zero reflection [1]. This result is related to the more general problem of constructing the potential from the phase shift and/or bound state energies in one or three dimensions, a problem which is of continuing interest [2-6].

In the present work we obtain the exact transmission amplitude for the class of multiparameter reflectionless index profiles discussed by Kay and Moses [1]. The exact result available in standard literature [2] are particular cases of the general index profiles described here. In this communication we extend the scope of this 
result to include the general case involving a set of $2 N$ real positive parameters. It is found that the exact phase is still expressible in terms of simple trigonometric functions and that it still exhibits a sort of additivity.

The eikonal approximation (EA) $[7,8]$, sometimes also referred to as the high energy approximation, is known to be a good approximation for the elastic scattering of a plane wave of wave number $k$ by a potential $V(x)$ of range $a$, if $|V(x)| / k^{2} \ll 1$ and $k a \gg 1$. By drawing an analogy between a potential and a refractive index $n(x)$ this approximation has been successfully adapted to suit a wide variety of optical scattering problems [8]. Since the reflection is ignored in the EA, the transmission probability is exact in this approximation. The difference with the exact result appears only in the phase. We find that this phase can be evaluated in the EA as well. Each term in the EA phase is noted to correspond to a term in the exact phase. It is observed that in the limit $k a \rightarrow \infty$, the EA phase tends to the exact phase term by term.

This paper is organized as follows. In Sec. 2 we begin by defining our notation and recapitulating some results of Kay and Moses [1]. In Sec. 3 we obtain the prescription for writing down the exact phase of the transmission amplitude. Comparison of the exact phase with the EA phase is discussed in Sec. 4. Finally, in Sec. 5 we conclude by summarizing our results.

\section{Preliminaries}

We will be considering a medium with refractive index $n(x)(-\infty<x<\infty)$, varying only in $x$-direction and a plane wave of wave number $k$ incident on it from the left. The problem then is effectively one-dimensional. Formal resemblance of the Helmholtz equation

$$
\frac{\mathrm{d}^{2} \psi(x)}{\mathrm{d} x^{2}}+k^{2} n^{2}(x) \psi(x)=0
$$

and the Schrödinger equation

$$
\frac{\mathrm{d}^{2} \psi(x)}{\mathrm{d} x^{2}}+\left[k^{2}-V(x)\right] \psi(x)=0
$$

leads to the relation $V(x)=k^{2}\left[1-n^{2}(x)\right]$. In the following we shall use the terms potential and index profile interchangeably.

Let us now summarize the results obtained in Ref. [1]. It was shown there that the Schrödinger equation (2) has an exact solution

$$
u(x, k)=\left(1+\sum_{n=1}^{N} \frac{f_{n}(x) \exp \left(k_{n} x\right)}{k_{n}+\mathrm{i} k}\right) \exp (\mathrm{i} k x),
$$

provided that the functions $f_{n}(x)$ satisfy

$$
\begin{array}{r}
A_{n} \exp \left(k_{n} x\right) \sum_{\nu=1}^{N} \frac{\exp \left(k_{\nu} x\right)}{k_{n}+k_{\nu}} f_{\nu}(x)+f_{n}(x)+A_{n} \exp \left(k_{n} x\right)=0 \\
n=1, \ldots, N
\end{array}
$$

The quantities $A_{n}$ and $k_{n}(n=1,2, \ldots, N)$ are arbitrary positive constants. Considering the differential operators

$$
L=\frac{\mathrm{d}^{2}}{\mathrm{~d} x^{2}}+\left[k^{2}-V(x)\right]
$$


and

$$
M_{n}=\frac{\mathrm{d}^{2}}{\mathrm{~d} x^{2}}-\left[k_{n}^{2}+V(x)\right]
$$

one can see by substitution from (3) that $L u=0$ provided

$$
\begin{aligned}
& M_{n} f_{n}(x)=0 \\
& V(x)=2 \frac{\mathrm{d}}{\mathrm{d} x} \sum_{n=1}^{N} f_{n}(x) \exp \left(k_{n} x\right) .
\end{aligned}
$$

Applying $M_{n}$ to the left hand side of Eq. (4) one gets a homogeneous system of linear algebraic equations for the quantities $M_{n} f_{n}(x)$ whose coefficient matrix has an inverse. The solution is therefore $M_{n} f_{n}(x)=0$, which means that if the $f_{n}(x)$ are determined by Eq. (4), it will automatically guarantee that they are the bound state eigenfunctions with energies $-k_{n}^{2}$. Furthermore, it was shown that $V(x)$ is negative for finite $x$ and approaches zero exponentially as $x$ approaches $\pm \infty$. The asymptotic form of $u(x, k)$ is now easily seen to be

$$
\begin{aligned}
& \lim _{x \rightarrow-\infty} u(x, k)=\exp (\mathrm{i} k x), \\
& \lim _{x \rightarrow+\infty} u(x, k)=\left(1+\sum_{n=1}^{N} \frac{g_{n}}{k_{n}+\mathrm{i} k}\right) \exp (\mathrm{i} k x),
\end{aligned}
$$

where we have written the asymptotic form of $f_{n}(x)$ as $g_{n} \exp \left(-k_{n} x\right)$ for positive $x$. The reflection amplitude is thus seen to be zero and the transmission amplitude

$$
t(k)=1+\sum_{n=1}^{N} \frac{g_{n}}{k_{n}+\mathrm{i} k}
$$

is therefore of unit modulus. We will see that $t(k)$ is independent of the constants $A_{n}$, i.e., it is a function of $k_{n}$ alone. The construction given by Eqs. (3) and (4) stems out of an earlier paper of Kay and Moses [9] where the search for a suitable function $V(x)$ was carried out through an integral equation technique and the demand that the complex reflection coefficient is zero.

\section{Exact phase shift}

Our purpose here will be to write $t(k)$ in a form suitable for the extraction of the phase. In some special situations, using the factorization method one can obtain explicit expressions for the exact solution and therefore $t(k)$. For example, for the potential [2] $V(x)=N\left(N+1\left(\operatorname{sech}^{2} x, t(k)\right.\right.$ is given by $\prod_{m=1}^{N} \frac{\mathrm{i} k+m}{\mathrm{i} k-m}$. Here, of course, the potential and the exact solution are given only implicitly in terms of the bound state wave functions which in turn are determined by the set of Eqs. (4). When only a few parameters $\left(k_{i}\right)$ are involved one can solve Eqs. (4) by matrix inversion and determine $f_{i}(x)$. For example, in the simplest case of one set of parameters $\left(k_{1}, A_{1}\right)$, Eq. (4) yields

$$
f_{1}(x)=-\frac{A_{1} \exp \left(k_{1} x\right)}{1+\left[A_{1} \exp \left(2 k_{1} x\right) / 2 k_{1}\right]}
$$


and consequently $t(k)$ is given by

$$
t(k) \equiv \lim _{x \rightarrow \infty}\left[1+\frac{f_{1}(x) \exp \left(k_{1} x\right)}{k_{1}+\mathrm{i} k}\right]=\frac{k+\mathrm{i} k_{1}}{k-\mathrm{i} k_{1}}
$$

Therefore, the phase $\phi$ is simply $2 \tan ^{-1}\left(k_{1} / k\right)$. In a similar manner, it is not very difficult to see that in the cases involving two or three parameter sets $t(k)$ assumes forms

and

$$
t(k)=\left[\left(k+\mathrm{i} k_{1}\right)\left(k+\mathrm{i} k_{2}\right)\right] /\left[\left(k-\mathrm{i} k_{1}\right)\left(k-\mathrm{i} k_{2}\right)\right]
$$

$$
t(k)=\left[\left(k+\mathrm{i} k_{1}\right)\left(k+\mathrm{i} k_{2}\right)\left(k+\mathrm{i} k_{3}\right)\right] /\left[\left(k-\mathrm{i} k_{1}\right)\left(k-\mathrm{i} k_{2}\right)\left(k-\mathrm{i} k_{3}\right)\right]
$$

respectively, so that the corresponding phases turn out to be additive in terms involving the parameters viz. $\phi(k)=2 \sum_{i=1}^{2} \tan ^{-1}\left(k_{i} / k\right)$ and $\phi(k)=$ $2 \sum_{i=1}^{3} \tan ^{-1}\left(k_{i} / k\right)$. We will now show that in the general $N$-parameter case $\left(k_{1} \ldots k_{n}\right), t(k)$ is given by

$$
t(k)=\prod_{n=1}^{N} \frac{k+\mathrm{i} k_{n}}{k-\mathrm{i} k_{n}}
$$

The right hand side of Eq. (9) is a meromorphic function with precisely $N$ simple poles at $k=\mathrm{i} k_{n}$ and $N$ simple zeros at $k=-\mathrm{i} k_{n}(n=1, \ldots, N)$. We can therefore use a theorem [10] which enables one to expand a meromorphic function into a sum of the principal parts of its Laurent expansions at all finite poles and an entire function. Thus,

$$
\prod_{n=1}^{N} \frac{k+\mathrm{i} k_{n}}{k-\mathrm{i} k_{n}}=\sum_{n=1}^{N} \frac{h_{n}}{k-\mathrm{i} k_{n}}+h(k),
$$

where $h(n)$ 's are the residues at the poles and $h(k)$ is an entire function whose value at infinity is seen to be one. Hence, by Liouville theorem, $h(k)$ is constant with value 1 everywhere. We now compare the series

$$
\prod_{n=1}^{N} \frac{k+\mathrm{i} k_{n}}{k-\mathrm{i} k_{n}}=1+\sum_{n=1}^{N} \frac{h_{n}}{k-\mathrm{i} k_{n}}
$$

with the series given by $(8)$ for $t(k)$ and note that the two series are identical if we can prove that $g_{n}=\mathrm{i} h_{n}$. Since Eq. (11) has zeros at $k=-\mathrm{i} k_{m}$ we find

$$
1+\mathrm{i} \sum_{n=1}^{N} \frac{h_{n}}{k_{m}+k_{n}}=0, \quad m=1, \ldots, N,
$$

whose solution is uniquely given by

$$
h_{n}=-\mathrm{i} \sum_{n}\left(M^{-1}\right)_{n m}
$$

with the real symmetric matrix $M$ defined by $M_{i j}=\frac{1}{k_{i}+k_{j}}$. On the other hand, the $g_{n}$ are determined by Eq. (4). Multiplying the $n$-th equation in (4) by $\exp \left(-k_{n} x\right)$ and allowing $x \rightarrow \infty$ we find

$$
1+\sum_{\nu} \frac{g_{n}}{k_{\nu}+k_{n}}=0, \quad n=1, \ldots, N .
$$


We see that $g_{n}$ and $\mathrm{i} h_{n}$ are unique solutions of the same equation and therefore they are equal. It remains to show that the matrix $M$ has non-zero determinant and therefore has an inverse. We introduce a matrix

$$
\widetilde{M}_{m n}=\frac{\mathrm{e}^{\left(k_{m}+k_{n}\right) x}}{k_{m}+k_{n}}
$$

so that $M_{m n}=\left.\widetilde{M}_{m n}\right|_{x=0}$. Let $y_{n}(n=1, \ldots, N)$ be a set of real numbers. Then

$$
\begin{gathered}
\sum_{m, n} y_{m} \widetilde{M} y_{n}=\int_{-\infty}^{x} \sum_{m, n} y_{m} \exp \left[\left(k_{m}+k_{n}\right) x\right] y_{n} \mathrm{~d} x \\
=\int_{-\infty}^{x}\left|\sum_{m} y_{m} \mathrm{e}^{k_{m} x}\right|^{2} \mathrm{~d} x \geq 0
\end{gathered}
$$

for all $x$. The equality sign holds only if all the $y_{n}$ are zero. The matrix $\widetilde{M}$ and therefore $M$ also has no eigenvector with zero eigenvalue and hence $M^{-1}$ exists. Equation (9) holds in general.

From (9) one immediately finds the exact phase to be

$$
\phi=2 \sum_{n=1}^{N} \tan ^{-1}\left(k_{n} / k\right) \text {. }
$$

A sort of additivity may be observed here, i.e., $\phi_{N+1}=\phi_{N}+\tan ^{-1}\left(k_{N+1} / k\right), \phi_{N}$ being the exact phase for the profile with $N$ parameters $k_{n}$. At present the significance of this additivity is not clear to us.

\section{The eikonal approximation}

In the EA the phase shift is a linear functional of the potential. It is given by the formula $[7,8]$

$$
\phi_{\mathrm{EA}}=-(1 / 2 k) \int_{-\infty}^{\infty} V(x) \mathrm{d} x,
$$

which with the help of Eq. (6b) can be expressed as

$$
\phi_{\mathrm{EA}}=-\left.(1 / k) \sum_{n=1}^{N} f_{n}(x) \exp \left(k_{n} x\right)\right|_{x=\infty}=-(1 / k) \sum_{n=1}^{N} g_{n} .
$$

To find $\sum_{n} g_{n}$, we use the formula

$$
\prod_{n=1}^{N} \frac{k+\mathrm{i} k_{n}}{k-\mathrm{i} k_{n}}=1+\sum_{n=1}^{N} \frac{g_{n}}{k_{n}+\mathrm{i} k} \text {. }
$$

Multiplying both sides by $\prod_{n=1}^{N}\left(k-\mathrm{i} k_{n}\right)$ and equating the coefficient of $k^{N-1}$ we get

$$
\sum_{n} g_{n}=-2 \sum_{n} k_{n}
$$

Thus $\phi_{\mathrm{EA}}$ becomes

$$
\phi_{\mathrm{EA}}=2 \sum_{n=1}^{N}\left(k_{n} / k\right) \text {. }
$$


This phase may now be contrasted with the exact phase (14). Clearly the EA can be viewed as a $k_{1} / k \rightarrow 0$ approximation for such dielectrics. Here it is assumed that $k_{1}>k_{2}>k_{3} \ldots k_{N}>0$.

To understand the meaning of the limit $k_{1} / k \rightarrow 0$ more clearly, let us turn our attention to the nature of the index profile. The index profile for $N=1$ is given by [1],

$$
n^{2}(x)=1+2\left(k_{1}^{2} / k^{2}\right) \operatorname{sech}^{2} k_{1}\left(x-x_{0}\right),
$$

where $k_{1}$ is related to the range $a_{1}$ of the potential via the relation $a_{1}=1 / k_{1}$. Defining $\alpha_{1}=k a_{1}$ as the size parameter, the refractive index at $x=x_{0}$ is given by

$$
\left[n^{2}\left(x_{0}\right)-1\right]=2\left(k_{1}^{2} / k^{2}\right)=2 / \alpha_{1}^{2} .
$$

Clearly the EA can be viewed here either as $\alpha_{1} \rightarrow \infty$ approximation or $\left|n\left(x_{0}\right)-1\right| \rightarrow 0$ approximation. It may be noted that the index profile (17) is a special case of the general index profile [11],

$$
n^{2}(x)=1+\frac{\lambda(\lambda-1) k_{1}^{2}}{k^{2}} \operatorname{sech}^{2} k_{1}\left(x-x_{0}\right),
$$

which becomes reflectionless for integer values of $\lambda$. The validity of the EA for the profile (19) requires two independent conditions because of the presence of strength variable $\lambda$.

As the phase in the EA is a linear functional of the potential, the result (16) suggests that in the $N$-parameter case the potential may be looked upon as a superposition of $N$ one-parameter potentials. But clearly this is true within the EA only.

\section{Conclusions}

For a multiparameter dielectric of the class considered here, we find that:

(i) the exact phase is expressible in terms of simple trigonometric functions. This phase is found to exhibit a sort of additivity. Each extra parameter $k_{n}$ in the potential introduces only one additional term, $2 \tan ^{-1}\left(k_{n} / k\right)$, in the phase. In this paper parameters of the index profile are not restricted to natural numbers.

(ii) A comparison of the EA phase with the exact phase shows that the EA may be interpreted either as high frequency or, what amounts to the same thing, a long-range approximation, $\left(k / k_{1}, \ldots, k / k_{n}\right) \rightarrow \infty$ approximation, or as $|n(x)-1| \rightarrow 0$ approximation.

(iii) In this validity domain of the $\mathrm{EA}$, the $N$-parameter reflectionless potential may obviously be viewed as a superposition of $N$ one-parameter reflectionless potentials.

\section{Acknowledgment}

We thank Dr. Avijit Lahiri for useful discussions. Detailed suggestions regarding improvements of the manuscript from the referee are gratefully acknowledged. 


\section{References}

[1] I. Kay, H.E. Moses, J. Appl. Phys. 27, 1503 (1956).

[2] R.K. Dodd, J.C. Eilbeck, J.D. Gibbon, H.C. Morris, Solitons and Nonlinear Wave Equations, Academic Press, London 1982.

[3] M.V. Berry, Proc. R. Soc. A 414, 31 (1987).

[4] M.V. Berry, C.J. Howls, J. Phys. A, Math. Gen. 23, L243 (1990).

[5] M. Lassaut, R.J. Lombard, Z. Pys. A 341, 125 (1992).

[6] K. Chadan, P.C. Sabatier, Inverse Problems in Quantum Scattering Theory, Springer, Berlin 1989.

[7] R.J. Glauber, Lectures in Theoretical Physics, Vol. 1, Eds. W.E. Brittin, L.G. Dunham, Interscience, New York 1959, p. 315.

[8] S.K. Sharma, D.J. Somerford, Nuovo Cimento D 12, 719 (1990) and references therein.

[9] I. Kay, H.E. Moses, Nuovo Cimento, Ser. X 3, 276 (1956).

[10] A.I. Markushevich, The Theory of Analytic Functions, Hindustan Publishing Corp., India 1963, p. 239 (translated from Kratkii Kurs Teorii Analiticheskikh Funktsii, Fizmatgiz, Moskva 1961, in Russian).

[11] S. Flugge, Practical Quantum Mechanics, Vol. 1, Springer-Verlag, Berlin 1971, p. 94. 